\title{
Effective Treatment of Vulvar-Vaginal Complaints in Women Taking Combined Oral Contraceptive with a New Medical Device in Gel: A Pilot Study
}

\author{
Filippo Murina, Franco Vicariotto \\ Lower Genital Tract Disease Unit, V. Buzzi Hospital, University of Milan, Milan, Italy \\ Email: filippomurina@tin.it
}

How to cite this paper: Murina, F. and Vicariotto, F. (2019) Effective Treatment of Vulvar-Vaginal Complaints in Women Taking Combined Oral Contraceptive with a New Medical Device in Gel: A Pilot Study. Open Journal of Obstetrics and Gynecology, 9, 326-333.

https://doi.org/10.4236/ojog.2019.93033

Received: January 7, 2019

Accepted: March 3, 2019

Published: March 6, 2019

Copyright $\odot 2019$ by author(s) and Scientific Research Publishing Inc. This work is licensed under the Creative Commons Attribution International License (CC BY 4.0).

http://creativecommons.org/licenses/by/4.0/

\begin{abstract}
Objective: To assess efficacy and tolerability of a new medical device in gel based on the synergic combination of Nioskin ${ }^{\text {tw }}$ Red Clover Extract noisome (NRC) and SylTech ${ }^{\mathrm{m}}$ system (SB), a complex of silicium microcrystals covalently bound with silver ions associated with hyaluronic acid to the treatment of vulvo-vaginal dryness and dyspareunia in women taking combined oral contraceptive (COC). Methods: Forty eight women were treated for 12 weeks with NRC + SB vaginal gel ( $0.75 \mathrm{~g} /$ day) for 3 weeks. After therapy, symptoms (dyspareunia and dryness) were evaluated through a 10 -cm VAS. Visual examination of the vagina and vulvar vestibule was also conducted, which included observations for petechiae, pallor, friability, dryness, and redness in the mucosa. Ratings were based on a 4-point scale ( 0 , none; 1 , mild; 2 , moderate; 3 , severe). Results: NRC + SB vaginal gel appears to be effective for relief vulvo-vagianl dryness and dyspareunia and an improvement of vaginal and vestibular trophism was noted. Conclusion: NRC $+\mathrm{SB}$ vaginal gel proved good treatment options for relief of vulvovaginal symptoms in women who take COC.
\end{abstract}

\section{Keywords}

Vaginal Dryness, Dyspareunia, Oral Contraceptive, Red Clover

\section{Introduction}

Vaginal dryness is a common symptom usually associated with the genitourinary syndrome of menopause as a result of low levels of estrogen. However, vaginal dryness may occur also in premenopausal women because of prolonged 
periods of low estrogens, such as low-dose combined oral contraceptive (COC) and/or progestogen-only methods [1].

In addition, very few studies on dyspareunia related to vaginal dryness have focused on location and quality of the pain. When we recognize, for example, the postmenopausal dyspareunia, it was reported that $95 \%$ of women also had localized provoked pain in the vestibule, despite the use of hormone supplements in $31 \%$ of them [2]. This is principally related to differences in nerve density between vagina and vulvar vestibule. A rich nerve plexus within the vaginal submucosa was identified, but it is composed only of sympathetic and parasympathetic axons with smaller contributions by sensory fibers; in the vulvar vestibule, the sensory nerve endings are dense and shallow, making this region physiologically more sensitive [3].

It was demonstrated that premenopausal women between 35 and 49 years of age reporting vaginal dryness could be also described as reduced vaginal lubrication during sexual arousal occurring, sometime or often, in around one third of the sample [4].

Poor arousal is strongly related to sexual pain and other female sexual dysfunction, but vaginal dryness may even interfere with simple activities such as walking or exercising, causing genital discomfort [5].

Many treatments (hormonal and non-hormonal) are available with the aim of restoring vaginal physiology and alleviating symptoms [6].

Women usually use commercial vaginal moisturizers on a regular basis to nourish tissues, whereas lubricants on demand may help to relieve vaginal dryness during coital activity. These are mainly a combination of protectants and thickening agents in a water-soluble base and are primarily used to relieve vaginal dryness during intercourse.

Hormonal replacement therapy (HRT), via systemic or topical routes, is widely used for the treatment of menopausal effects on urogenital tract. All local vaginal estrogen products have been recognized as being effective and well tolerated for treating vaginal atrophy [6].

In recent years, phytoestrogen supplements have become attractive as safer alternatives, and their efficacy has been investigated in experimental and clinical trials [7].

The efficacy of isoflavone vaginal gel for the treatment and management of symptoms of vaginal estrogen deficiency-induced vaginal atrophy in postmenopausal women was confirmed in a recent trial [8], but no data are available regarding the use of topical phytoestrogen in premenopausal women with vulvar and vaginal dryness. The aim of the current investigation was to evaluate the efficacy of a new medical device in gel based on the synergic combination of $\mathrm{Ni}$ oskin $^{\text {Tw }}$ Red Clover Extract noisome (NRC), a unique ultra-deformable vesicles effectively delivering the concentrated isoflavones aglycones of Red Clover and SylTech $^{\text {tw }}$ system (SB), a complex of silicium microcrystals covalently bound with silver ions associated with hyaluronic acid, in the supportive treatment of vulvo-vaginal dryness and dyspareunia in women taking COC. 


\section{Methods}

\subsection{Patients}

Inclusion criteria were: Caucasian childbearing potential women aged 18 50-year-old using a COC as a reliable method of contraception since at least 3 months and reporting subjective vaginal dryness and dyspareunia. Exclusion criteria were: being pregnant or lactating women or women of childbearing potential, not using a reliable contraceptive method or perimenopausal women, having had previous episodes of vaginal bleeding or inter-menstrual spotting in the last 6 months, being diagnosed with urinary or vaginal infections; having used in the last 15 days before inclusion in the study any kind of lubricants and/or hormonal and/or non-hormonal products for local treatment of vaginal dryness and dyspareunia and having an identified or presumptive hypersensitivity to gel ingredients.

\subsection{Study Design}

This observational clinical study was performed in accordance with the Declaration of Helsinki and International Standards of Good Clinical Practice (ICH-E6). Study subjects were instructed about the design of the study and had to provide a written informed consent, before undergoing a screening visit, and the local ethical committee approved the study. Eligible participants received a $1 \mathrm{mg}$ gel containing NRC and SB in single-dose vaginal dispenser/applicator. Patients were instructed to apply gel internally in vagina (about 2/3) and on the vulvar vestibule (about $1 / 3$ ) daily for 3 weeks $(0.75 \mathrm{~g}$ ), at bedtime in the evening, after intimate cleaning. Overall treatment duration was about 23 days; vaginal gel was applied between two menstrual/withdrawal bleedings (6th \pm 1 to 27 th \pm 1 day of the menstrual cycle).

\subsection{Assessment}

All women underwent medical examination (interview and gynecologic examination), in order to determine patient eligibility. During the visits at 30 days, patients reported by a questionnaire any symptoms of vaginal dryness and dyspareunia, considered as primary endpoint of the study. Participants recorded intensity of symptoms using a 10-cm VAS. The scale's left extremity indicates the complete absence of symptoms (0) and the right extremity indicates the worst possible symptom, and women rated dyspareunia and dryness from 0 to 10.Secondary endpoints were the differences from baseline to the end of the study of visual examination of the vagina and vulvar vestibule, which included observations for petechiae, pallor, friability, dryness, and redness in the mucosa. Ratings were based on a 4-point scale ( 0 , none; 1 , mild; 2 , moderate; 3 , severe). Other endpoints were: 1) proportion of subjects withdrawing from the study due to low tolerability or low efficacy of the gel; 2) time to withdrawal from the study, for the cases defined as above; 3) global efficacy and tolerability, as eva- 
luated by both the subject and by the investigator at the end of the study; 4) incidence of adverse events (AEs). Statistical analyses (Wilcoxon Mann-Whitney test-Kruskal-Wallis test) were performed by comparing endometrial thickness and changes in scores between baseline and weeks 4 and 12 for each symptom and sign. Data were expressed as means and $95 \%$ confidence intervals (CIs), accompany $\mathrm{P}$ values for all the endpoints.

\section{Results}

Of the 51 women enrolled in the study, 48 completed the treatment. Three patients discontinued the studies prematurely the study for personal reasons unrelated to the use of vaginal gel. Table 1 showed baseline characteristics of the study sample.

In the efficacy primary analysis population, an overall decrease of symptoms was observed after the treatment. Such advantage was confirmed by the analysis of changes, which showed a highly statistically significant difference at the end of the study treatment $(\mathrm{p}<0.003)$. The sub-analysis on vaginal and vestibular dryness mean changes showed a highly statistically significant difference in favour of gel treatment, and the same was true for dyspareunia (Figure 1). Similarly, the clinical success rates calculated upon the difference in proportions between symptoms after the treatment was about $65 \%$ and $51 \%$ for vaginal dryness and dyspareunia respectively. Statistically significant improvement of vestibular and vaginal trophism were also found after the treatment (Figure 2). The single item changes of vestibular trophism from baseline to the end of the study were more marked than vaginal objectivity.

No severe or serious AEs were observed; five subjects (9.8\%) reported an initial light burning at application, symptom that disappears with the treatment naturally.

Table 1. Patients demographics and baseline characteristics.

\begin{tabular}{ll}
\hline & Patients $(\mathrm{n}=51)$ \\
\hline Age (y) & $30.1( \pm 5.6)$ \\
Range & $26-33$ \\
Contraceptive composition (n. women) & \\
-EE 20 mcg + DSP 3 mg & 29 \\
-EE 30 mcg + DSP 3 mg & 14 \\
-EE 30 mcg + DNG 2 mg & 8 \\
Symptoms (VAS) and Signs* & \\
Dyspareunia & $6.3( \pm 4.6)$ \\
Vestibular/vaginal dryness & $7.2( \pm 5.2)$ \\
Vestibular trophism* & $10.1( \pm 6.6)$ \\
Vaginal trophism & $5.2( \pm 3.8)$ \\
\hline
\end{tabular}

$\mathrm{EE}=$ ethinyl estradiol; DSP = drospirenone; DNG: dienogest; ${ }^{*}$ Scored using a 4-point scale of petechiae, pallor, friability, dryness, and redness in the mucosa. (0, none; 1 , mild; 2 , moderate; 3 , severe). Data are presented as the mean values \pm standard deviation. 


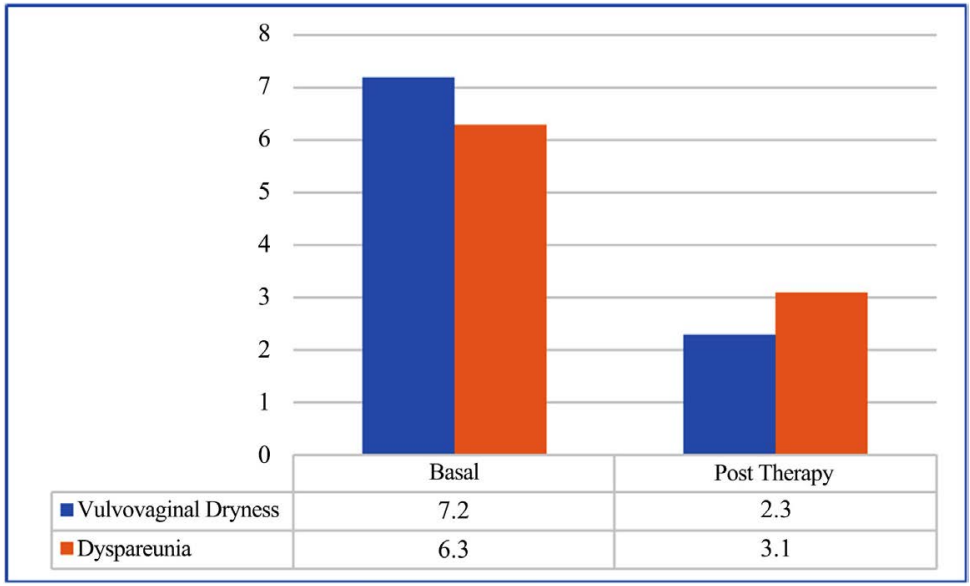

Figure 1. Symptoms after therapy. Data of VAS presented as the mean $(\mathrm{p}<0.003)$.

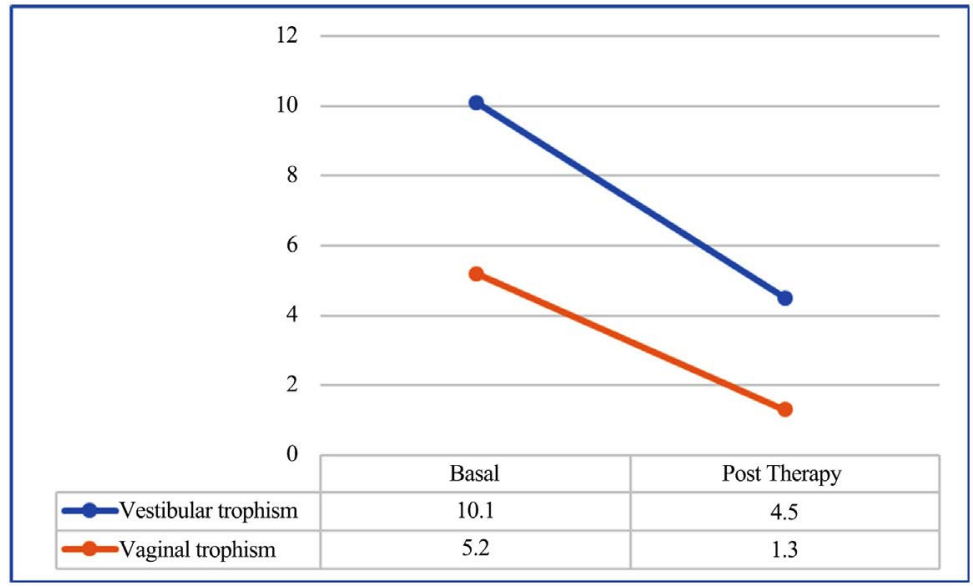

Figure 2. Vestibular and vaginal trophic score at baseline and week 12. Data are expressed as score using a 4-point scale of petechiae, pallor, friability, dryness, and redness in the mucosa $(0$, none; 1 , mild; 2 , moderate; 3 , severe).

\section{Discussion}

This observational study demonstrates that daily use of vaginal gel based on $\mathrm{Ni}$ oskin $^{\text {mu }}$ Red Clover Extract noisome (NRC) is an effective well-tolerated and safe treatment of vaginal dryness and other associated symptoms related to the use of COC.

After about 3-week treatment, there was a significant improvement in symptoms of vaginal discomfort, and subjective improvement corresponds to an amelioration of lower genital tract health assessed by vaginal and vestibular trophism score.

A vast range of topical products, both lubricants and moisturizers, has been tested in women reporting vaginal dryness mainly due to estrogen depletion with menopause of any kind [9]. Very few data are available in premenopausal women, even though clinical experience indicates that they mostly use lubricants to fix transient symptoms of vaginal discomfort, especially during coital activity [10]. Furthermore, women using COC were significantly more likely to report 
decreased vaginal lubrication [11].

The use of a hormonal method was associated with a higher proportion of sexual events involving pain and discomfort during sex, which is consistent with other research that has shown a relationship between prolonged use of combined oral contraceptives with experiences of pain in the vulvar vestibule [12] [13]. Given the importance of taking hormonal contraception for fertility control and extra-contraceptive benefits, our data on the efficacy of NRC + SB vaginal gel in relieving vaginal and vestibular dryness and other associated symptoms are of great interest for healthcare practitioners.

Several studies have found that COC increase the relative risk of developing pain in the vulvar vestibule (vestibulodynia) [14]. In addition to alterations in serum hormone (estradiol and testosterone) and Sex Hormone Binding Globulin (SHBG) levels, COC has been shown to induce changes in hormone receptors as well as alter the morphological pattern in the vestibular mucosa, making it thin and weak [15].

Therefore, the lack of vaginal and vestibular lubrication enhances friction and with the thinning of epithelial layers increases the risk of micro-trauma resulting in pain enhancement. In this study we used a new medical device in gel based on Nioskin $^{\text {tw }}$ Red Clover Extract noisome (NRC). This is a unique ultra-deformable vesicle effectively delivering the concentrated isoflavones aglycones of Red Clover allowing obtaining an elevated bioavailability of the drug. A strength of the trial is the synergic combination SylTech ${ }^{\text {tx }}$ system (SB), a complex of silicium microcrystals covalently bound with silver ions associated with hyaluronic acid. Siltech $^{\text {tw }}$ microcrystals, the micro-technology ensuring a range of particles size averaging of 0.2 - 0.3 micron, achieve the maximum extent of interaction on tissue creating a protective film layer [16]. Another strength of our study was also the vestibular application of gel by combining estrogen-like agonist effect of

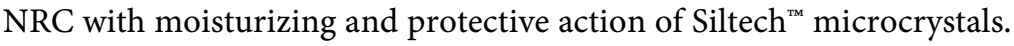

Our data provide a more nuanced understanding of hormonal contraception use and its effects on a woman's sexual experiences by examining associations between hormonal contraceptive use and measures of dyspareunia. Based on the assessment of treatment and symptom severity in this study, NRC + SB vaginal gel was shown to be effective for relieving both moderate and intense symptoms, particularly vaginal dryness complaints and dyspareunia, mirroring the effects of estrogen use. One limitation of this study is the absence of follow-up, but the therapy may be continued for as long as the patients are distressed by their symptoms.

Further, prospective research with diverse women starting various methods of contraception is needed to enhance understanding of the potential negative sexual side effects of contraceptive methods, their prevalence, and possible mechanisms.

\section{Funding}

This study was supported by the Associazione Italiana Vulvodinia, a non-profit 
Italian association whose mission is to improve the health and quality of life of women experiencing vulvodynia and chronic vulvar pain.

\section{Conflicts of Interest}

The authors declare no conflicts of interest regarding the publication of this paper.

\section{References}

[1] Burrows, L.J., Basha, M. and Goldstein, A.T. (2012) The Effects of Hormonal Contraceptives on Female Sexuality: A Review. Journal of Sexual Medicine, 9, 2213-2223. https://doi.org/10.1111/j.1743-6109.2012.02848.x

[2] Kao, A., Binik, Y.M., Amsel, R., Funaro, D., Deroux, N. and Khalife, S. (2012) Biopsychosocial Predictors of Postmenopausal Dyspareunia: The Role of Steroid Hormones, Vulvovaginal Atrophy, Cognitive-Emotional Factors, and Dyadic Adjustment. Journal of Sexual Medicine, 9, 2066-2076. https://doi.org/10.1111/j.1743-6109.2012.02771.x

[3] Goldstein, I., Meston, C., Davis, S. and Traish, A. (2005) Women's Sexual Function and Dysfunction: Study Diagnosis and Treatment. CRC Press, Boca Raton, Florida. https://doi.org/10.1201/b14618

[4] Bachmann, G. (1995) Urogenital Ageing: An Old Problem Newly Recognized. Maturitas, 22, S1-S5. https://doi.org/10.1016/0378-5122(95)00956-6

[5] Nappi, R.E. and Kokot-Kierepa, M. (2012) Vaginal Health: Insights, Views \& Attitudes (VIVA): Results from an International Survey. Climacteric, 15, 36-44. https://doi.org/10.3109/13697137.2011.647840

[6] Gandhi, J., Chen, A., Dagur, G., et al. (2016) Genitourinary Syndrome of Menopause: An Overview of Clinical Manifestations, Pathophysiology, Etiology, Evaluation, and Management. American Journal of Obstetrics and Gynecology, 215, 704-711. https://doi.org/10.1016/j.ajog.2016.07.045

[7] Nikander, E., Rutanen, E.M. and Nieminen, P. (2005) Lack of Effect of Isoflavonoids on the Vagina and Endometrium in Postmenopausal Women. Fertility and Sterility, 83, 137-142. https://doi.org/10.1016/j.fertnstert.2004.09.003

[8] Lima, S.M., Yamada, S.S., Reis, B.F., Postigo, S., Galvão da Silva, M.A. and Aoki, T. (2013) Effective Treatment of Vaginal Atrophy with Isoflavone Vaginal Gel. Maturitas, 74, 252-258. https://doi.org/10.1016/j.maturitas.2012.11.012

[9] Sinha, A. and Ewies, A.A. (2013) Non-Hormonal Topical Treatment of Vulvovaginal Atrophy: An Up-to-Date Overview. Climacteric, 16, 305-312. https://doi.org/10.3109/13697137.2012.756466

[10] Sutton, K.S., Boyer, S.C., Goldfinger, C., Ezer, P. and Pukall, C.F. (2012) To Lube or Not to Lube: Experiences and Perceptions of Lubricant Use in Women with and without Dyspareunia. Journal of Sexual Medicine, 9, 240-250. https://doi.org/10.1111/j.1743-6109.2011.02543.x

[11] Burrows, L.J., Basha, M. and Goldstein, A.T. (2012) The Effects of Hormonal Contraceptives on Female Sexuality: A Review. Journal of Sexual Medicine, 9, 2213-2223. https://doi.org/10.1111/j.1743-6109.2012.02848.x

[12] Battaglia, C., Battaglia, B., Mancini, F., Busacchi, P., Paganotto, M.C., Morotti, E. and Venturoli, S. (2012) Sexualbehavior and Oralcontraception: A Pilot Study. Journal of Sexual Medicine, 9, 550-557. https://doi.org/10.1111/j.1743-6109.2011.02597.x 
[13] Smith, N.K., Jozkowski, K.N. and Sanders, S.A. (2014) Hormonal Contraception and Female Pain, Orgasm and Sexual Pleasure. Journal of Sexual Medicine, 11, 462-470. https://doi.org/10.1111/jsm.12409

[14] Greenstein, A., Ben-Aroya, Z., Fass, O., Militscher, I., Roslik, Y., Chen, J. and Abramov, L. (2007) Vulvar Vestibulitis Syndrome and Estrogen Dose of Oral Contraceptive Pills. Journal of Sexual Medicine, 4, 1679-1683. https://doi.org/10.1111/j.1743-6109.2007.00621.x

[15] Johannesson, U., Sahlin, L., Masironi, B., Hilliges, M., Blomgren, B., Rylander, E. and Bohm-Starke, N. (2008) Steroid Receptor Expression and Morphology in Provoked Vestibulodynia. American Journal of Obstetrics and Gynecology, 198, 311.e1-6. https://doi.org/10.1016/j.ajog.2007.09.041

[16] SiltehTM Micro Silver Micro System. http://www.linnea.ch 